\title{
The microbiological signature of human cutaneous leishmaniasis lesions exhibits restricted bacterial diversity compared to healthy skin
}

\author{
Vanessa R Salgado1, Artur TL de Queiroz ${ }^{1,2}$, Sabri S Sanabaniं ${ }^{3}$, Camila I de Oliveira ${ }^{1,2}$, \\ Edgar M Carvalho ${ }^{1,4,5}$, Jackson ML Costa1, Manoel Barral-Netto ${ }^{1,2}$, Aldina Barral ${ }^{1,2} /+$

\begin{abstract}
${ }^{1}$ Fundação Oswaldo Cruz, Centro de Pesquisas Gonçalo Moniz, Salvador, BA, Brasil ²Instituto de Investigação em Imunologia, São Paulo, SP, Brasil ${ }^{3}$ Faculdade de Medicina da Universidade de São Paulo, Hospital das Clínicas, Departamento de Patologia, São Paulo, SP, Brasil ${ }^{4}$ Universidade Federal da Bahia, Hospital Universitário Prof Edgard Santos, Serviço de Imunologia, Salvador, BA, Brasil ${ }^{5}$ Instituto Nacional de Ciência e Tecnologia em Doenças Tropicais, Salvador, BA, Brasil
\end{abstract}

Localised cutaneous leishmaniasis (LCL) is the most common form of cutaneous leishmaniasis characterised by single or multiple painless chronic ulcers, which commonly presents with secondary bacterial infection. Previous culture-based studies have found staphylococci, streptococci, and opportunistic pathogenic bacteria in LCL lesions, but there have been no comparisons to normal skin. In addition, this approach has strong bias for determining bacterial composition. The present study tested the hypothesis that bacterial communities in LCL lesions differ from those found on healthy skin (HS). Using a high throughput amplicon sequencing approach, which allows for better populational evaluation due to greater depth coverage and the Quantitative Insights Into Microbial Ecology pipeline, we compared the microbiological signature of LCL lesions with that of contralateral HS from the same individuals. Streptococcus, Staphylococcus, Fusobacterium and other strict or facultative anaerobic bacteria composed the LCL microbiome. Aerobic and facultative anaerobic bacteria found in HS, including environmental bacteria, were significantly decreased in LCL lesions $(p<0.01)$. This paper presents the first comprehensive microbiome identification from LCL lesions with next generation sequence methodology and shows a marked reduction of bacterial diversity in the lesions.

Key words: microbiome - LCL - wound - healthy skin - high-throughput sequencing

Cutaneous leishmaniasis (CL) is broadly divided into three major clinical phenotypes: diffuse CL (DCL), presenting multiple nonulcerative nodules, mucosal leishmaniasis (LM), characterised by destructive mucosal inflammation, and localised CL (LCL), the most frequent manifestation of CL, characterised by single or multiple painless chronic ulcerative skin lesions, developing at the site of the infected sandfly bite (Reithinger et al. 2007, Costa et al. 2009). CL in the New World is caused mainly by Leishmania (Viannia) braziliensis, Leishmania (Viannia) guyanensis, Leishmania (Leishmania) amazonensis, and Leishmania (Leishmania) mexicana (Grimaldi Jr et al. 1989). In the Northeast Region of Brazil, LCL and LM are predominantly caused by $L$. $(V$.) braziliensis (Rosa et al. 1988) and DCL is most commonly caused by L. amazonensis (Bittencourt et al. 1989).

LCL ulcers usually present with a slow recovery and may last several months or years in the absence of antiparasitic treatment (Salman 1999). Therapy is broadly ac-

doi: 10.1590/0074-02760150436

Financial support: CNPq (479717/2011-7), NIH (AI088650), iii-

INCT, INCT-D

VRS was supported by fellowships from CNPq and CAPES.

VRS and ATLQ contributed equally to this work.

+Corresponding author: abarral@bahia.fiocruz.br

Received 25 November 2015

Accepted 1 March 2016 complished with pentavalent antimonials (Glucantime ${ }^{\circledR}$; Sanofi-Aventis, Brazil) recommended as first line drugs. Therapeutic response to these drugs is usually favourable, presenting variable cure rates (from 60-100\%) (Azeredo-Coutinho et al. 2007). The ulcer chronicity, constant environmental exposure, and poor hygiene at the lesion site associated with ground proximity in lesions located in lower limbs, one of the most affected site, may promote polymicrobial infections. Secondary bacterial infections are frequently observed in LCL patients. This condition, in addition to being uncomfortable for the patient, may sometimes impair the healing process of ulcer and require antibiotic treatment (Vera et al. 2002, 2006, Fontes et al. 2005, Gonçalves et al. 2009).

Prejudicial effects on the healing process have been reported in the presence of a complex bacterial colonisation also in diabetic lesions and of other chronic wounds (Daltrey et al. 1981, Halbert et al. 1992, Davies et al. 2004, Grice et al. 2010, Percival et al. 2010, Scales \& Huffnagle 2013). In LCL lesions, it was observed that individuals presenting secondary infection with purulent secretion and harbouring concomitant infections with Streptococcus pyogenes, Staphylococcus aureus, Pseudomonas aeruginosa, Morganella morganii, and Enterococcus durans presented with a delayed healing process (Isaac-Márquez \& Lezama-Dávila 2003). In other study, patients presenting secondary bacterial infection in CL lesions presented lower cure rates to systemic treatment with glucantime compared with patients who did not presenting contamination. Authors suggest decreased effect of glucantime in CL lesions complicated with secondary bacterial infection. S. aureus was the most preva- 
lent organism in these contaminated lesions (Sadeghian et al. 2011). The delayed time in CL lesion healing requires repeated treatment cycles, with harmful effects associated with drug toxicity (Mitropoulos et al. 2010).

Naik et al. (2012) evaluated the role of skin microbiota on local immunity using germ-free (GF) and specific pathogen free (SPF) mice in a model of dermal infection induced by $L$. major. GF mice infected intradermally with $L$. major showed smaller lesions with reduced oedema and necrosis compared to SPF mice, however with higher parasite load in the lesion. These outcomes were associated with a smaller production of interferon-gamma and tumour necrosis factor-alpha by cutaneous T-cell and demonstrated the role of skin microbiota in Leishmania infection control. Previous studies comparing GF and conventional (microbiota-bearing) mice infected subcutaneously with $L$. major (revised by Lopes et al. 2016) demonstrated GF mice failed to heal lesions and presented higher parasite load at the infection site than conventional mice. The cytokine production profile did not differ in both; however macrophages from GF mice were not as efficient at killing parasites as conventional mice, suggesting an important role of microbiota in macrophage activation (Oliveira et al. 2005). Vieira et al. (1998) also found similar results with GF mice failing to resolve lesions after 13 weeks of $L$. major infection, in contrast with the conventional mice. Despite this, the cytokine profiles were indistinguishable in both groups. GF mice macrophages, albeit capable of producing nitric oxide (NO) in response to leishmanial infection, were no able to destroy the parasites. Absence of the normal indigenous microbiota was suggested as cause of increased susceptibility to L. major infection (Vieira et al. 1998).

However, the leishmaniasis microbiome-composition in humans remains unclear. Few studies for bacterial community identification in LCL lesions have been performed. Previous culture-based studies reported Staphylococcus spp, Streptococcus spp, Enterococcus spp, Pseudomonas spp, and other opportunistic bacteria in LCL lesions (Vera et al. 2006, Shirazi et al. 2007, Ziaei et al. 2008, Gonçalves et al. 2009). Nevertheless, this culture-based methodology has a strong bias for estimation of bacterial composition since bacteria presenting low relative abundance, fastidious and/or "unculturable" organisms are underestimated (Rhoads et al. 2012, Scales \& Huffnagle 2013).

Advanced molecular methods have indicated that only about $1 \%$ or $2 \%$ of all the skin-colonising bacteria could be cultivated under usual conditions (Gontcharova et al. 2010, Bertesteanu et al. 2014). Furthermore, culture-independent tests revealed a much greater diversity in skin bacterial communities compared to culture-based estimations (Grice \& Segre 2011, Rhoads et al. 2012, Scales \& Huffnagle 2013). The use of massive molecular methods allows deep insights into the microbiome-composition in general and also in the LCL microbiome because it is more sensitive than culture, as described for other chronic wounds (Rhoads et al. 2012). Our hypothesis is that bacterial communities in LCL lesions differ from those on contralateral healthy skin
(HS). Thus, our aim was to characterise the LCL lesion microbiome and to compare it to that found on HS, in the same individuals, using next generation sequencing.

\section{SUBJECTS, MATERIALS AND METHODS}

Patient characterisation - The median age from subjects was 35 years (range 20-48 years). Subjects were two women and eight men without significant differences related to gender. Individuals chosen for the study lived in the endemic area an average of 32.3 years (range 8-44 years), most had agricultural activity, and presented ulcerated skin lesions clinically consistent with CL (Costa et al. 2009). Patients were confirmed with CL after positive results in, at least, one of the following tests realised to the inclusion of them in the study: delayedtype hypersensitivity (DTH) with Leishmania antigen, parasite isolation in culture, parasite identification in histopathological studies, or a positive L. braziliensis polymerase chain reaction (PCR) performed, as previously described (Weirather et al. 2011). No patient had received previous treatment for leishmaniasis or antibiotics in the last month before sample collection.

Wound samples and HS collection - Samples were collected in The Health Post of Corte de Pedra, state of Bahia, Brazil, a CL endemic area. Twenty paired swab samples (10 samples collected from LCL lesions and 10 samples collected from contralateral HS) were obtained using sterile technique after wound cleaning with saline and debridement, when a crust was present. The material collected in swab samples was transferred to MO BIO PowerBead Tubes (PowerSoil DNA Isolation kit; MO BIO Laboratories, Inc, USA) and immediately frozen at $-80^{\circ} \mathrm{C}$ until DNA isolation.

Management of patients - After sample collection, patients were treated with intravenous pentavalent antimony $\mathrm{Sb}^{\mathrm{V}}(20 \mathrm{mg} / \mathrm{kg} / \mathrm{day}$ for 20 days). The lesion outcome was observed up to the healing. Patients who had failure of treatment after the first course and the lesion persisted after 90 days after initiation of treatment received a second course of $\mathrm{Sb}^{\mathrm{V}}(20 \mathrm{mg} / \mathrm{kg} /$ day for 20 days $)$. A third $\mathrm{Sb}^{\mathrm{V}}$ course in combination with pentoxifylline (400 mg/kg/3 times daily) was administrated to patients who had persistence of the lesion 60 days after initiation of the second $\mathrm{Sb}^{\mathrm{V}}$ course (Machado et al. 2002).

LCL lesion and HS sample DNA isolation - Bacterial DNA isolation was performed according to the PowerSoil DNA Isolation Kit (MO BIO) manufacturer's instructions. DNA amounts were measured using the Qubit Quant-iT dsDNA BR Assay Kit protocol (Life Technologies, USA). Laminar flows and reagents used in DNA isolation were treated with ultraviolet before use to reduce the risk of contamination. DNA was submitted to V4 16S rRNA PCR amplification as described below.

16S rRNA barcode library preparation and sequencing - For each patient, two different amplicon libraries were prepared: one from LCL ulcers and the other from HS. The hypervariable V4 region of the 16S rRNA gene from each sample was amplified in triplicate us- 
ing a primer set (515f/806r), as previously described (Caporaso et al. 2011, 2012). Amplification reactions were performed using the FastStart High Fidelity PCR System (Roche Applied Science, Germany) with a final volume of $25 \mu \mathrm{L}$ containing $1.8 \mathrm{mM} \mathrm{MgCl}, 200 \mu \mathrm{M}$ dNTP, $0.4 \mu \mathrm{M}$ primer (515f and 806r), 2.5 U FastStart High Fidelity Enzyme Blend, and a $100 \mathrm{ng} / \mu \mathrm{L}$ DNA sample. The PCR conditions were as follows: an initial step of $3 \mathrm{~min}$ at $94^{\circ} \mathrm{C}, 35$ cycles consisting of $45 \mathrm{~s}$ at $94^{\circ} \mathrm{C}, 60 \mathrm{~s}$ at $50^{\circ} \mathrm{C}$, and $90 \mathrm{~s}$ at $72^{\circ} \mathrm{C}$, and a final step of $10 \mathrm{~min}$ at $72^{\circ} \mathrm{C}$. Amplicons were visualised using $1.5 \%$ agarose gels and ethidium bromide nucleic acid gel stain in a $0.5 x$ tris-borate-ethylenediamine tetraacetic acid buffer. To confirm that the PCR reagents were not source of bacterial sequences, no-template control PCR was performed. No visible amplification signal was observed in this negative control on a gel, indicating that bacterial contamination was minimal.

The triplicate PCR products were pooled and purified using the PureLink PCR Purification Kit (Life Technologies), according to the manufacturer's instructions. Following purification, each library was normalised to ensure equal library representation in pooled samples. The final libraries were pooled at equimolar concentration, denatured, and mixed with PhiX control to increase sequence diversity. Finally, the prepared library was loaded onto an Illumina MiSeq clamshell style cartridge for sequencing.

Processing and analysis of $16 \mathrm{~S} r \mathrm{RNA}$ sequences - After sequencing, all 16 rRNA raw sequence data were demultiplexed, quality-filtered, and analysed using Quantitative Insights Into Microbial Ecology (QIIME) v.1.7.0 pipeline (Kuczynski et al. 2012). After assignment of sequences, primer and tag sequences were removed before operational taxonomic unit (OTU) clustering. OTU clustering was performed with a $97 \%$ similarity threshold. Taxonomic identities were assigned using the RDP classifier tool (v.10.28) with 0.97 confidence threshold using Greengenes Database (from May 2013). Different numbers of reads per sample were obtained. Therefore, the sequence data were rarefied at 400,000 sequences per sample to account for this variation for Chao and observed species index calculations (i.e., alpha diversity), and principal coordinates analysis (PCoA) was performed using the phylogeny-based unweighted UniFrac distance metric (i.e., beta diversity).

Statistical analyses - Identification of OTUs that were significantly different in abundance in LCL lesions and contralateral HS was performed by paired $t$ test with Bonferroni correction. To determine whether any groups of samples contained significantly different bacterial communities, analysis of similarities (ANOSIM) was conducted. Using the unweighted UniFrac distance matrix, distances were grouped as "within group" or "between group". Significance levels were calculated by comparing the $\mathrm{R}$ statistic against the distribution generated from 10,000 permutations of the randomised dataset. The data were entered into a custom database (Excel, Microsoft Corp) and analysed using Prism 5. Quantitative data were reported as mean \pm standard deviation or median (1st and 3rd quartile). Categorical data were reported as percentage and $95 \%$ confidence inter- val. Within patient phylum and genus level differences between lesions and HS were compared using multiple Wilcoxon rank tests for paired data. A p-value less than 0.05 were considered to be statistically significant.

Availability of supporting data - Raw sequences were deposited in the National Center for Biotechnology Information Short Read Archive (ncbi.nlm.nih.gov/ sra) and are available with the following accessions: SAMN03198271, SAMN03198273, SAMN03198274, SAMN03198275, SAMN03198276, SAMN03198277, SAMN03198314, SAMN03198315, SAMN03198316, and SAMN03198317.

Ethics - The Gonçalo Moniz Research Centre/Oswaldo Cruz Foundation Ethical Committee approved the study (IRB 000026120; CEP 339/2010). The Declaration of Helsinki protocols were followed and all subjects provided written consent prior to participation in the study.

\section{RESULTS}

Cohort description - To evaluate bacterial communities in LCL lesions, 10 subjects presenting ulcerated skin lesions with circular contours, infiltrative borders painless, and background with crude granules, epidemiologically and clinically consistent with LCL (Costa et al. 2009), were included in the study. One patient showed multiple lesions located mainly in the lower limbs, while the other nine patients presented single ulcers at different body sites shown in Table. These sites are the most frequent associated to LCL lesions in our experience (data not shown). Table also reported the demographic data, clinical characteristics, results of Leishmania diagnosis tests, and LCL lesion outcome for the patients included in the study.

All subjects presented positive results in the DTH test with Leishmania antigen (Table). Parasites were isolated in culture from patients 3 and 5. Parasites were also detected in histopathological exams of patients 6 and 7. All patients, except 3, 7, and 10, tested positive in the L. braziliensis PCR (Weirather et al. 2011). Patient 10 was the only one that the direct tests (culture, histopathology, or PCR) were negative. However, this patient presented an epidemiological history compatible with leishmaniasis (living for 39 years in the endemic area in an agricultural working), positive results in DTH test, and multiple lesions in lower limbs clinically very characteristic of LCL.

In relation to the lesions outcome after treatment, seven patients had complete regression of the lesion after the first $\mathrm{Sb}^{\mathrm{V}}$ course (Table). Three patients (5, 9, and 10) showed treatment failure after the first $\mathrm{Sb}^{\mathrm{V}}$ course and received a second course of $\mathrm{Sb}^{\mathrm{V}}$. Only for patient 10 was necessary a third $\mathrm{Sb}^{\mathrm{V}}$ course in combination with pentoxifylline. All patients showed complete lesion regression after treatment, consistent with leishmaniasis infection.

Analysis of 16S rRNA sequences - To evaluate whether bacterial communities in LCL lesions differed from those found on HS, samples from 10 different laboratorial and clinical confirmed LCL lesions and from the corresponding contralateral HS of the same patient were sequenced using a high throughput amplicon sequencing methodology. Demultiplexed 16 rRNA raw sequence 


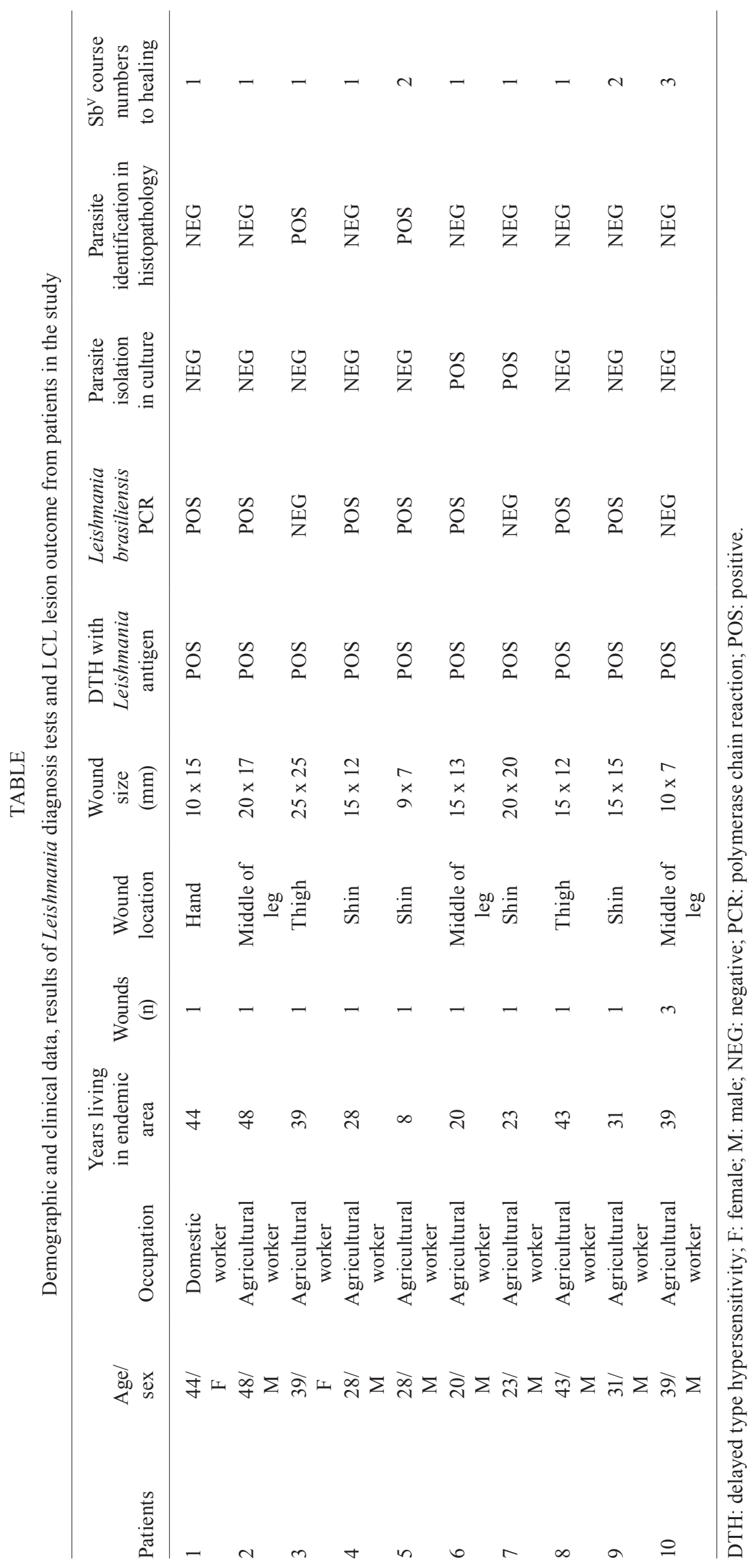



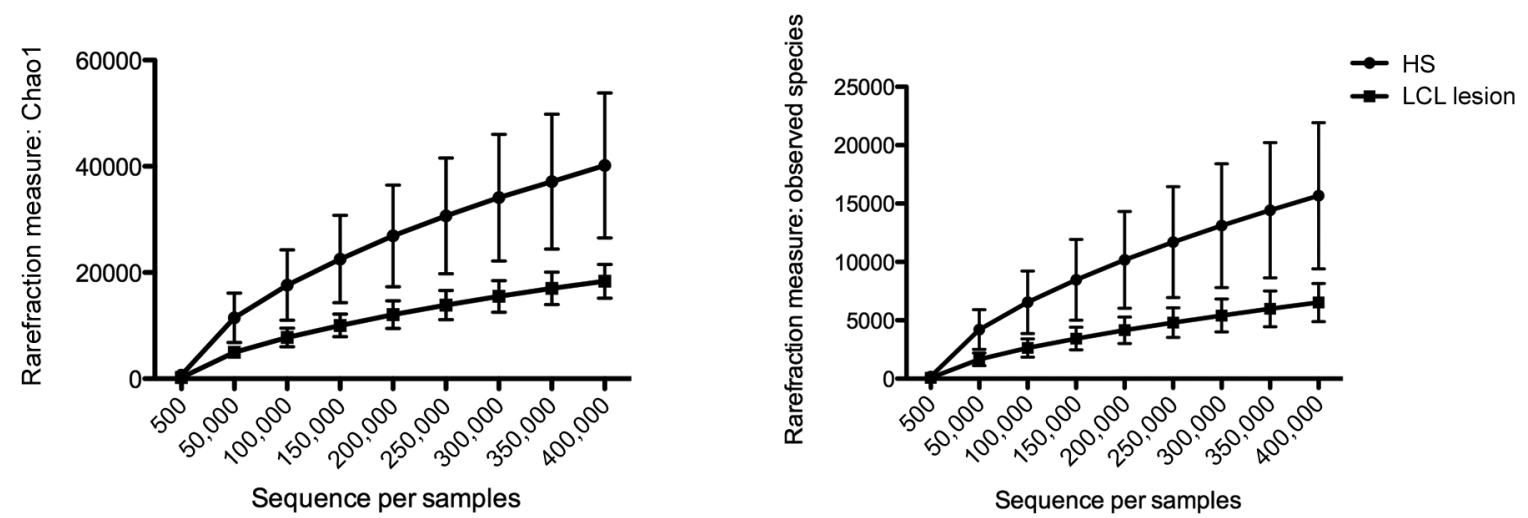

Fig. 1: rarefaction analysis for the Chao index (A) and observed species (B) of 16S rRNA sequences obtained from localised cutaneous leishmaniasis (LCL) lesions (square) and contralateral healthy skin (HS) (sphere). Lines represent the average of each group while the error bars represent the standard deviations.

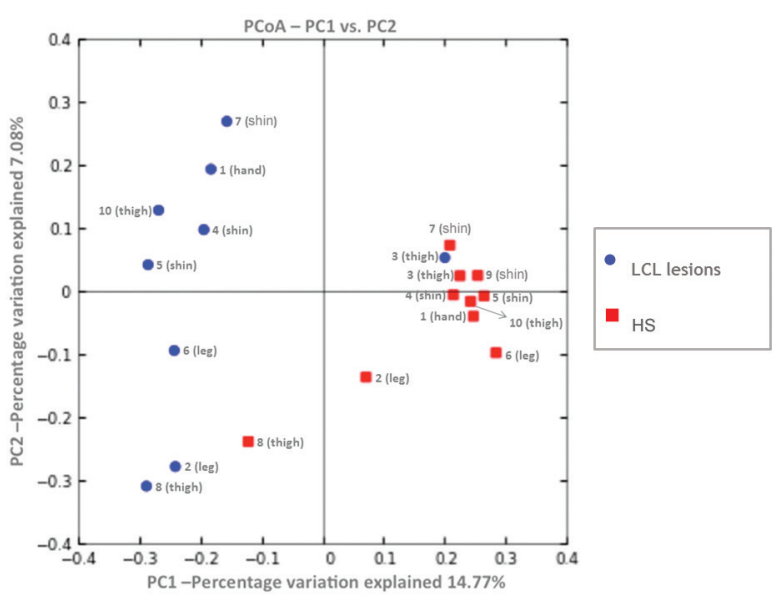

Fig. 2: principal coordinates analysis (PCoA) of unweighted UniFrac distances between localised cutaneous leishmaniasis (LCL) lesions (blue) and contralateral healthy skin (HS) (red) samples. The percentage of variation explained by each PCoA is indicated on the axis.

data were quality-filtered and analysed using QIIME. After the quality filter checks, 13,759,042 high quality reads were obtained: 7,823,305 reads were from HS and $5,935,737$ reads from LCL lesions. The mean sequence number per sample was 687,952.1 $\pm 101,984$ (range from $408,767-927,648)$. The average length of sequences was $251 \mathrm{bp}$. Thirty-one phyla and 862 OTUs were identified in Greengenes Database. The lesion sample reads from patient 9 were excluded from statistical analysis because almost $90 \%$ of sequences from this patient were not classified into any phyla or OTU.

Alpha and beta diversity - Rarefaction measurements (alpha diversity), shown in Fig. 1, demonstrated significant differences in observed species and Chao indices between the HS microbiome vs. LCL lesions denoting that HS presented significantly higher diversity levels compared to LCL wounds $(\mathrm{p}<0.01)$.
PCoA plots based on unweighted UniFrac distance matrix (beta diversity), shown in Fig. 2, exhibits separation between most HS and LCL lesion samples (ANOSIM, R $=0.58, p=0.002)$ indicating that the LCL lesion microbiome differs from HS. PCoA also suggests that HS (red) has more samples with similar bacterial composition to each other than the LCL lesion. In addition, PCoA showed a mild cluster of samples of HS (red) related to the body sites where these samples were collected. Shin, thigh, and leg were grouped near to other samples from the same site.

Differences in LCL lesions and HS microbial community at phylum level - Thirty-one bacterial phyla were detected in both LCL lesions and HS. Most sequences detected in LCL lesions and HS were assigned to five phyla: Firmicutes, Actinobacteria, Proteobacteria, Fusobacteria, and Bacteroidetes. Chloroplast/Cyanobacteria were also detected in all HS samples and in LCL lesions from two patients. Fig. 3 shows the relative abundance of these phyla was variable considering the sampled skin sites from HS and LCL lesions. Unknown phyla in Fig. 3 represent sequences that have not been classified with the database used. This limitation occurs due the query insufficient alignment scores with the sequences database or low probability with RDP classifier.

Fig. 4 shows the cumulative percentages of sequences from bacterial phyla detected in HS and LCL lesions. The vast majority of sequences detected in LCL lesions were members of Firmicutes (54.3\%), Actinobacteria (11.7\%), Fusobacteria (11.6\%), Proteobacteria (8.7\%), and Bacteroidetes $(5.1 \%)$. Firmicutes were more frequent in LCL lesions $(54.3 \%)$ than HS (34.6\%). This phylum's abundance was significantly higher $(\mathrm{p}=0.02)$ in LCL lesions. Actinobacteria and Proteobacteria were detected in LCL lesions, however with significantly lower percentages compared to HS (HS $26.2 \%$ vs. $11.7 \%$ LCL and HS $21.8 \%$ vs. $8.7 \%$ LCL, respectively). Cyanobacteria showed very low percentages in LCL lesions $(0.1 \%)$ compared to HS $(2 \%)$. The presence of this phylum in HS also differed significantly from LCL lesions $(p=0.001)$. One of the major phyla detected in LCL lesions was Fusobacteria, however without statistical significance. Bacteroidetes showed low percentages in both sites (LCL $5.1 \%$ vs. $4.9 \%$ HS). 


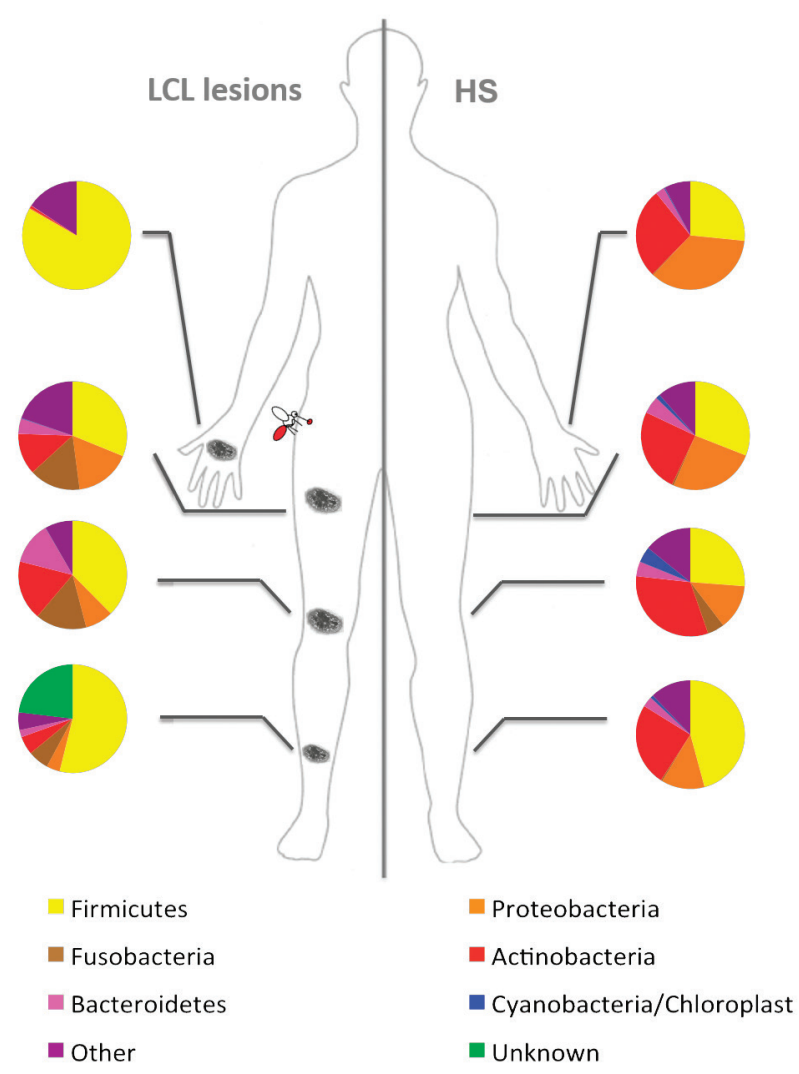

Fig. 3: topographical distribution of bacterial phyla detected in localised cutaneous leishmaniasis (LCL) lesions and healthy skin (HS) samples by high-throughput Illumina sequencing. Pie charts represent the major bacterial phyla found in different topographic body sites (hand, thigh, middle of leg, and shin).

Differences in LCL lesions and HS microbiota at genus level - About 862 bacterial genera were found in LCL lesions and HS, however the 33 genera demonstrated in Fig. 5 were the most common at both sites with relative abundances ranging the sampled skin sites (hand, middle leg, shin, and thigh) from HS and LCL. These were the bacterial genera which presented relative abundance greater than or equal to $2.5 \%$.

Fig. 6 shows the cumulative percent of bacterial sequences in HS and LCL lesions and also the significant differences found in LCL lesions and HS microbiome. Bacterial genera were separated by the $\mathrm{O} 2$ tolerance to facilitate the preview. Streptococcus was the only genus found significantly increased in LCL lesions compared to HS $(p=0.01)$. Although not statistically significant, LCL lesions showed increased levels of strict and/or facultative anaerobic bacteria compared to HS. Moreover, about 15 environmental bacterial genera were significantly decreased in LCL lesions compared to HS, as shown in Fig. 6.

Considering the LCL lesions microbiome, the ubiquitous genera were Staphylococcus (present in all patients), followed by Streptococcus (detected in 8 of 9 patients), Corynebacterium, and Peptoniphilus (found in 7 of 9

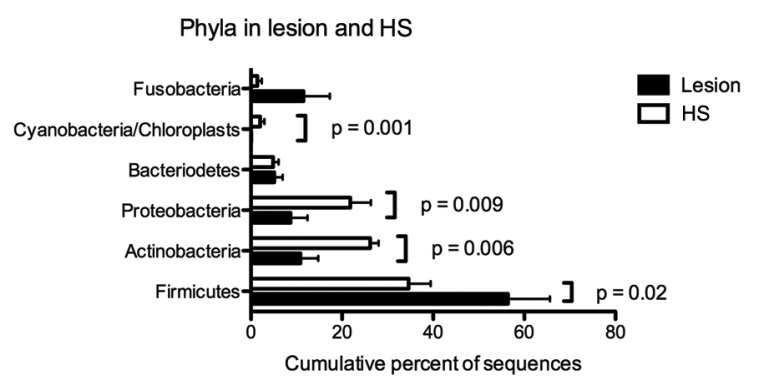

Fig. 4: cumulative percentages of sequences from bacterial phyla detected by high-throughput Illumina sequencing in healthy skin (HS) (white bars) and localised cutaneous leishmaniasis (LCL) lesion samples (dark bars) compared using multiple Wilcoxon rank tests for paired data.

patients), Peptostreptococcus, and Fusobacterium (observed in 6 and 5 patients, respectively). Staphylococcus, Streptococcus, and Fusobacterium were the predominant genera in lesion samples of patient 5 and 10, as observed in Fig. 5. These patients presented with a delayed healing process and took two and three cycles of leishmanicidal drugs, respectively, to complete the healing of the lesion.

\section{DISCUSSION}

We compare the microbiological composition of laboratorial and LCL lesions with the contralateral HS microbiome from the same individuals using high throughput amplicon sequencing approach, which allows for deeper and broader insight into microbiological diversity. Our results showed that cutaneous microbiological signatures in human leishmaniasis lesions from patients living in an area with high predominance of $L$. braziliensis transmission (up to 95\%) (Rosa et al. 1988) exhibit restricted bacterial diversity compared to those of HS. Three out of 10 patients did not show positive results in PCR. In these cases, it was not possible to identify the implicated Leishmania. Two of these patients did not show delayed healing, but presented parasites in histopathological examination (patient 3) and culture (patient 7). The negative results of patients 3 and 7 in the PCR could be explained by the presence of inhibitors in the isolated DNA of these patients, which hamper the amplification of target DNA in the PCR reaction. Different samples could also be used in the PCR and other tests (histology and culture) which caused this conflict in the results. Only patient 10 showed delayed healing and had negative results in the direct tests, suggesting that the PCR and other tests had insufficient sensitivity for this patient diagnosis. Although we did not identify other Leishmania species in these patients, it is likely they were infected with L. braziliensis or L. amazonensis. As only, these two species were identified in human cases from this endemic area. However, L. amazonensis is mainly associated with other clinical manifestation as DCL (Costa et al. 2009).

Both rarefaction measures and PCoA (alpha and beta diversity) results suggest that LCL and HS microbiome composition is different (Figs 1,2). This result shows that compositionally intact HS bacterial colonisation is differ- 


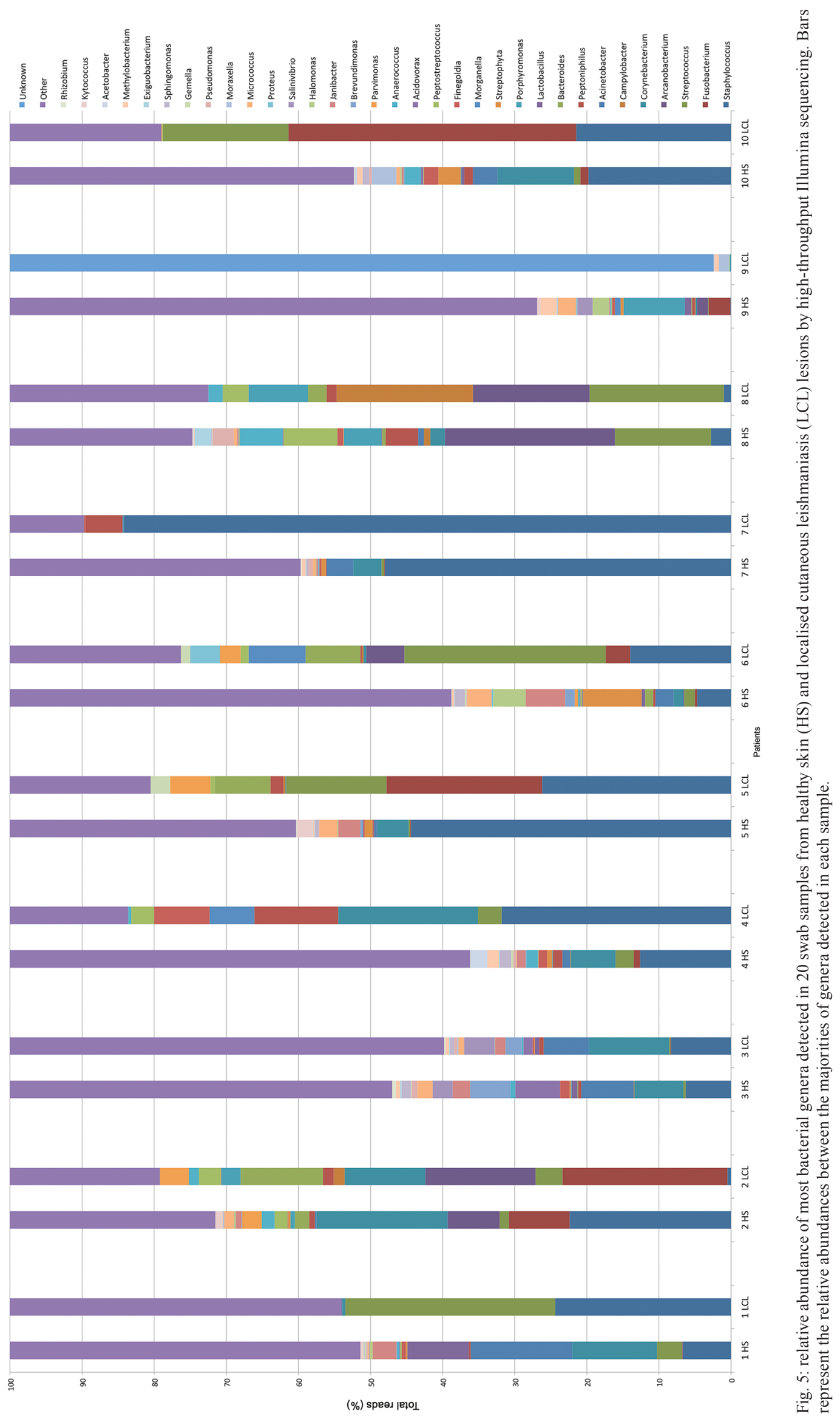


ent from the opened wound with compromised epidermis caused by Leishmania infection. This microbiome composition variation in LCL lesions is probably because an open wound with compromised epidermis, associated with a subjacent inflammation caused by Leishmania, provides unbalance in indigenous microbial components (Scales \& Huffnagle 2013). Our results suggest that the disturbance in LCL lesions is associated with an opportunistic contamination by commensal bacteria, which could resist to subjacent inflammation promoted by Leishmania, and develop against less adapted bacteria. In addition, this condition could provide pathogenic colonisation from different aetiologies (Scales \& Huffnagle 2013).

Moreover, PCoA shows HS has more samples with similar composition to each other than the LCL lesion, probably because patients have similar microbiota related to the topographical body sites (Figs 2, 3). This sitedependent grouping based on the bacterial composition in the skin has already been described (Grice et al. 2009, Kong \& Segre 2012).

The LCL microbiome showed lower diversity compared to HS. This restricted bacterial diversity in microbial community in affected compared to HS has also been reported in both inflammatory or not skin diseases, such as diabetic ulcers (Gontcharova et al. 2010), pso- riasis (Alekseyenko et al. 2013), and atopic dermatitis (Kong et al. 2012). High bacterial diversity is associated with host protection, by innate and adaptive immune system modulation (Grice et al. 2010, Grice \& Segre 2011, Kong \& Segre 2012). The inflammation triggered by the innate immune system provides a suitable environment to some commensal bacteria, which could resist to the subjacent inflammation promoted by Leishmania and hampering development of less adapted bacteria.

Another explanation for the bacterial diversity reduction in LCL is the competition and cooperation behaviour in mixed microbial population, as bacteriocin and virulence factor production, which are commonly described in interspecies bacterial competition (Scales \& Huffnagle 2013). Most adapted bacteria could influence development of another bacterium in a LCL environment.

We observed in HS samples a similar composition to the skin microbiome composed by the following phyla: Actinobacteria, Firmicutes, Bacteroidetes, and Proteobacteria (Grice \& Segre 2011). Despite being less frequent, Cyanobacteria and Fusobacteria were also observed, suggesting microbiome composition variation for patients from our endemic area. Concerning the LCL microbiome, a similar microbial community was found in the microbiome of nonhealing diabetic foot ulcers, with
A

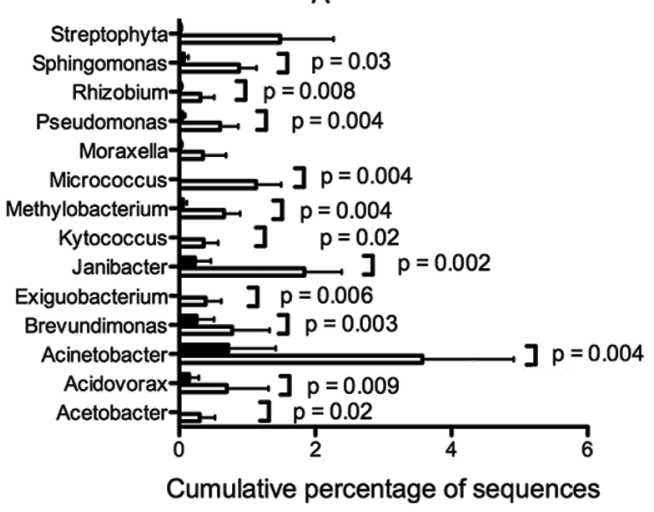

C

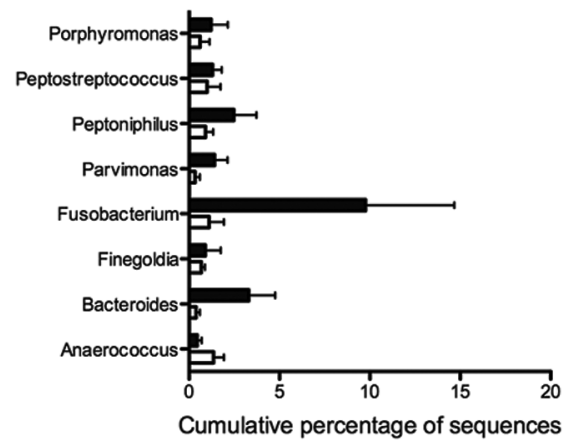

B

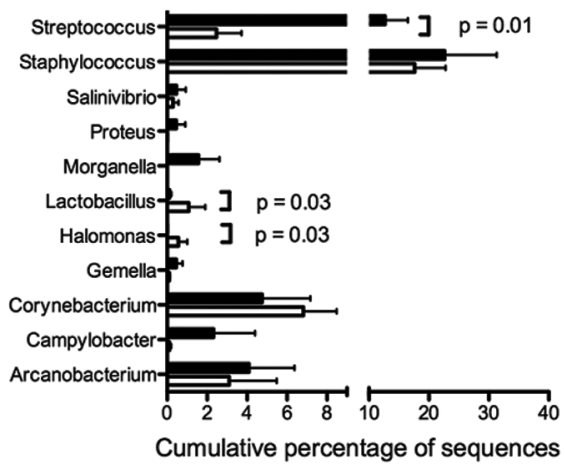

D

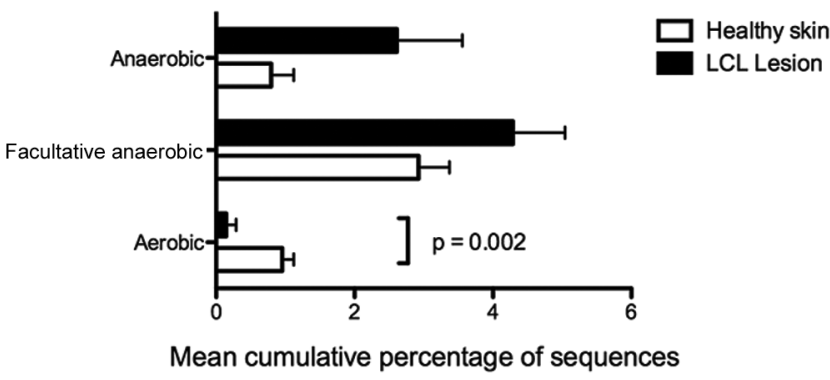

Fig. 6: bacterial genera detected by high-throughput Illumina sequencing in healthy skin (HS) (white bars) and localised cutaneous leishmaniasis (LCL) lesions (dark bars) samples compared using multiple Wilcoxon rank tests for paired data. A: cumulative percent of aerobic bacterial sequences in HS and LCL lesions; B: cumulative percent of anaerobic facultative and microaerophilic bacterial sequences in HS and LCL lesions; C: cumulative percent of strict anaerobic bacterial sequences in HS and LCL lesions; D: mean cumulative percent of sequences from anaerobic, facultative anaerobic/microaerophilic, and aerobic bacteria in HS and LCL. 
similar percentages of Firmicutes (67\%), Actinobacteria (14\%), Proteobacteria (9.8\%), Bacteroidetes $(7.3 \%)$, and Fusobacteria (1.4\%) at phylum level (Gardner et al. 2013).

Some aerobic bacteria presented decreased levels in LCL lesions. For instance, Lactobacillus and Pseudomonas play important protective roles on skin by the production of lactic acid, antimicrobial, and antifungal compounds, respectively (Cogen et al. 2008, Slover \& Danziger 2008). However, many other bacteria detected in our study were not previously associated with the skin microbiome, that could be associated to high read number (687,952 reads mean per sample), allowing identification of new microbiome components.

We observed that anaerobes (Fusobacterium, Bacteroides, and Peptoniphilus), microaerophiles, and facultative anaerobic (Streptococcus, Staphylococcus, Morganella, Campylobacter, and Arcanobacterium) bacteria were most frequently detected in LCL (Fig. 6), with a reduction of the aerobic bacteria in normal skin. Similar results were observed in chronic wounds microbiome from several aetiologies, which presented a significantly larger proportion of anaerobes (Bacteroidetes, Fusobacterium), large quantities of Gram-negative rods such as Pseudomonas, Proteus, E. coli, and Klebsiella, and an increased proportion of Staphylococcus and Streptococcus. Chronic wounds also had a noticeably decreased proportion of protective coloniser in normal skin (Han et al. 2011).

These anaerobic bacteria may survive in the oxygen presence at LCL lesions, as observed in other wounds also exposed to air, by the symbiotically association with aerobic species. These aerobic bacteria could consume oxygen, creating localised low oxygen niches, allowing obligate anaerobes maintaining (Dowd et al. 2008, Gontcharova et al. 2010). Somehow, the infectioninduced low oxygen microenvironment in LCL lesions could contribute with Leishmania persistence, once hypoxia negatively disturbs the killing of Leishmania due to type $2 \mathrm{NO}$ synthase activity reduction of macrophages (Das \& Lu 2014, Mahnke et al. 2014).

Analysing the differences in the skin microbiota at genus level, only Streptococcus was significantly higher in LCL lesion. This genus was previously linked to chronic ulcer infection (Issac-Márquez \& Lezama-Dávila 2003, Shirazi et al. 2007, Dowd et al. 2008, Wolcott et al. 2009, Gontcharova et al. 2010) and healing delay (Davies et al. 2004, Bertesteanu et al. 2014). This suggests an important role of Streptococcus in chronic wounds maintenance, as LCL lesions.

Interestingly, we observed in two of three patients (patients 5 and 10) with delayed healing (take 2 or $3 \mathrm{Sb}^{\mathrm{V}}$ cycles) an association of three bacterial genera Streptococcus, Staphylococcus, and Fusobacterium in a higher frequency (Fig. 5). The other patient with delayed healing was excluded from analysis due to sequencing issues. These three genera were found in HS samples from the same patients; however, Streptococcus and Fusobacterium showed a lower prevalence in HS and presented a high increase in LCL lesions. These species alone seem not to influence healing time as observed in patients 1 and 2. These findings suggest a synergistic effect of these three genera on the healing process of LCL lesions. This was an interesting observation in our study; however it must be confirmed by further studies due the small number of analysed patients with delayed healing.

Ours results show the high bacterial community diversity in HS and LCL lesions. These findings demonstrate that traditional evaluation by culturing pathogenic bacteria is highly biased. The significant bacterial diversity reduction in LCL lesion microbiomes shows predominance of Streptococcus and strict or facultative anaerobes. It was also possible observe that microbial profile changes were directly related to the delayed healing in two out of the 10 patients. In these patients, Streptococcus, Staphylococcus, and Fusobacterium concomitant infection were implicated in a complex bacterial formation, leading to delayed healing. The present results provide insights about the influence of these specific bacteria on healing time, although this observation needs more investigation, due the sample size. Future investigations with larger samples are required to improve the microbiome understanding at different body sites. Moreover, temporal analyses are necessary to monitoring the microbiome changes during the ulcer-healing course.

\section{ACKNOWLEDGEMENTS}

To the volunteers who participated in this study, to the personnel of the Corte de Pedra Health Post, for assistance with sample collection, to Regis Brandim Gomes, for the sandfly cartoon, and to the undergraduate students and scientific initiation fellows, Rodrigo Pessoa, Jaqueline T Watanabe, and Paula Calabria, for their help with DNA isolation and barcoded library preparation.

\section{REFERENCES}

Alekseyenko AV, Perez-Perez GI, de Souza A, Strober B, Gao Z, Bihan M, Li K, Methé BA, Blaser MJ 2013. Community differentiation of the cutaneous microbiota in psoriasis. Microbiome 1: 31 .

Azeredo-Coutinho RBG, Mendonça SCF, Callahan H, Portal AC, Grögl M 2007. Sensitivity of Leishmania braziliensis promastigotes to meglumine antimoniate (glucantime) is higher than that of other Leishmania species and correlates with response to therapy in American tegumentary leishmaniasis. J Parasitol 93: 688-693.

Bertesteanu S, Triaridis S, Stankovic M, Lazar V, Chifiriuc MC, Vlad M, Grigore R 2014. Polymicrobial wound infections: pathophysiology and current therapeutic approaches. Int J Pharm 463: 119-126.

Bittencourt A, Barral A, de Jesus AR, de Almeida RP, Grimaldi Jr G 1989. In situ identification of Leishmania amazonensis associated with diffuse cutaneous leishmaniasis in Bahia, Brazil. Mem Inst Oswaldo Cruz 84: 585-586.

Caporaso JG, Lauber CL, Walters WA, Berg-Lyons D, Huntley J, Fierer N, Owens SM, Betley J, Fraser L, Bauer M, Gormley N, Gilbert JA, Smith G, Knight R 2012. Ultra-high-throughput microbial community analysis on the Illumina HiSeq and MiSeq platforms. ISME J 6: 1621-1624.

Caporaso JG, Lauber CL, Walters WA, Berg-Lyons D, Lozupone CA, Turnbaugh PJ, Fierer N, Knight R 2011. Global patterns of 16S rRNA diversity at a depth of millions of sequences per sample. Proc Natl Acad Sci USA 108 (Suppl. 1): 4516-4522.

Cogen AL, Nizet V, Gallo RL 2008. Skin microbiota: a source of disease or defence? Br J Dermatol 158: 442-455.

Costa JML, Saldanha ACR, Nasciento D, Sampaio G, Carneiro F, Silva LM, Barral A 2009. Clinical modalities, diagnosis, and 
therapeutic approach of the tegumentary leishmaniasis in Brazil. Gaz Med Bahia 79 (Suppl. 3): 70-83.

Daltrey DC, Rhodes B, Chattwood JG 1981. Investigation into the microbial flora of healing and non-healing decubitus ulcers. $J$ Clin Pathol 34: 701-705.

Das LM, Lu KQ 2014. The virtues of oxygenation: low tissue oxygen adversely affects the killing of Leishmania. J Invest Dermatol 134: 2303-2305.

Davies CE, Hill KE, Wilson MJ, Stephens P, Hill CM, Harding KG, Thomas DW 2004. Use of $16 \mathrm{~S}$ ribosomal DNA PCR and denaturing gradient gel electrophoresis for analysis of the microfloras of healing and nonhealing chronic venous leg ulcers. J Clin Microbiol 42: 3549-3557.

Dowd SE, Wolcott RD, Sun Y, McKeehan T, Smith E, Rhoads D 2008. Polymicrobial nature of chronic diabetic foot ulcer biofilm infections determined using bacterial tag encoded FLX amplicon pyrosequencing (bTEFAP). PLoS ONE 3: e3326.

Fontes CO, Carvalho MAR, Nicoli JR, Hamdan JS, Mayrink W, Genaro O, Carmo LS, Farias LM 2005. Identification and antimicrobial susceptibility of micro-organisms recovered from cutaneous lesions of human American tegumentary leishmaniasis in Minas Gerais, Brazil. J Med Microbiol 54: 1071-1076.

Gardner SE, Hillis SL, Heilmann K, Segre JA, Grice EA 2013. The neuropathic diabetic foot ulcer microbiome is associated with clinical factors. Diabetes 62: 923-930.

Gonçalves EGR, dos Reis Filho SA, de Oliveira EG, Pareira ALN, da Silva AR, Costa JML 2009. Bacterial infection in cutaneous leishmaniasis: bacterial pattern and sensitivity to antibiotics. Rev Soc Bras Med Trop 42: 219-221.

Gontcharova V, Youn E, Sun Y, Wolcott RD, Dowd SE 2010. A comparison of bacterial composition in diabetic ulcers and contralateral intact skin. Open Microbiol J 4: 8-19.

Grice EA, Kong HH, Conlan S, Deming CB, Davis J, Young AC, NISC Comparative Sequencing Program, Bouffard GG, Blakesley RW, Murray PR, Green ED, Turner ML, Segre JA 2009. Topographical and temporal diversity of the human skin microbiome. Science 324: 1190-1192.

Grice EA, Segre JA 2011. The skin microbiome. Nat Rev Microbiol 9: 244-253.

Grice EA, Snitkin ES, Yockey LJ, Bermudez DM, Liechty KW, Segre JA 2010. Longitudinal shift in diabetic wound microbiota correlates with prolonged skin defense response. Proc Natl Acad Sci USA 107: 14799-14804.

Grimaldi Jr G, Tesh RB, McMahon-Pratt D 1989. A review of the geographic distribution and epidemiology of leishmaniasis in the New World. Am J Trop Med Hyg 41: 687-725.

Halbert AR, Stacey MC, Rohr JB, Jopp-McKay A 1992. The effect of bacterial colonization on venous ulcer healing. Australas J Dermatol 33: 75-80.

Han A, Zenilman JM, Melendez JH, Shirtliff ME, Agostinho A, James G, Stewart PS, Mongodin EF, Rao D, Rickard AH, Lazarus GS 2011. The importance of a multi-faceted approach to characterizing the microbial flora of chronic wounds. Wound Repair Regen 19: 532-541.

Isaac-Márquez AP, Lezama-Dávila CM 2003. Detection of pathogenic bacteria in skin lesions of patients with chiclero's ulcer. Reluctant response to antimonial treatment. Mem Inst Oswaldo Cruz 98: 1093-1095.

Kong HH, Oh J, Deming C, Conlan S, Grice EA, Beatson MA, Nomi$\cos$ E, Polley EC, Komarow HD, Mullikin J, Thomas J, Blakesley
R, Young A, Chu G, Ramsahoye C, Lovett S, Han J, Legaspi R, Sison C, Montemayor C, Gregory M, Hargrove A, Johnson T, Riebow N, Schmidt B, Novotny B, Gupta J, Benjamin B, Brooks S, Coleman H 2012. Temporal shifts in the skin microbiome associated with disease flares and treatment in children with atopic dermatitis. Genome Res 22: 850-859.

Kong HH, Segre JA 2012. Skin microbiome: looking back to move forward. J Invest Dermatol 132: 933-939.

Kuczynski J, Stombaugh J, Walters WA, González A, Caporaso JG, Knight R 2012. Using QIIME to analyze 16S rRNA gene sequences from microbial communities. Curr Protoc Microbiol 01: Unit-1E.5.

Lopes ME, Carneiro MB, dos Santos LM, Vieira LQ 2016. Indigenous microbiota and leishmaniasis. Parasite Immunol 38: 37-44.

Machado P, Araújo C, da Silva AT, Almeida RP, D’Oliveira Jr A, Bittencourt A, Carvalho EM 2002. Failure of early treatment of cutaneous leishmaniasis in preventing the development of an ulcer. Clin Infect Dis 34: E69-E73.

Mahnke A, Meier RJ, Schatz V, Hofmann J, Castiglione K, Schleicher U, Wolfbeis OS, Bogdan C, Jantsch J 2014. Hypoxia in Leishmania major skin lesions impairs the NO-dependent leishmanicidal activity of macrophages. J Invest Dermatol 134: 2339-2346.

Mitropoulos P, Konidas P, Durkin-Konidas M 2010. New World cutaneous leishmaniasis: updated review of current and future diagnosis and treatment. J Am Acad Dermatol 63: 309-322.

Naik S, Bouladoux N, Wilhelm C, Molloy MJ, Salcedo R, Kastenmuller W, Deming C, Quinones M, Koo L, Conlan S, Spencer S, Hall JA, Dzutsev A, Kong H, Campbell DJ, Trinchieri G, Segre JA, Belkaid Y 2012. Compartmentalized control of skin immunity by resident commensals. Science 337: 1115-1119.

Oliveira MR, Tafuri WL, Afonso LC, Oliveira MA, Nicoli JR, Vieira EC, Scott P, Melo MN, Vieira LQ 2005. Germ-free mice produce high levels of interferon- $\gamma$ in response to infection with Leishmania major but fail to heal lesions. Parasitology 131: 477-488.

Percival SL, Thomas JG, Williams DW 2010. Biofilms and bacterial imbalances in chronic wounds: anti-Koch. Int Wound J 7: 169-175.

Reithinger R, Dujardin J, Louzir H 2007. Cutaneous leishmaniasis. Lancet Infect Dis 7: 581-596.

Rhoads DD, Cox SB, Rees EJ, Sun Y, Wolcott RD 2012. Clinical identification of bacteria in human chronic wound infections: culturing vs. $16 \mathrm{~S}$ ribosomal DNA sequencing. BMC Infect Dis 12: 321.

Rosa AC, Cuba CC, Vexenat A, Barreto AC, Marsden PD 1988. Predominance of Leishmania braziliensis in the regions of Três Braços and Corte de Pedra, Bahia, Brazil. Trans R Soc Trop Med Hyg 82: 409-410.

Sadeghian G, Ziaei H, Bidabadi LS, Baghbaderani AZ 2011. Decreased effect of glucantime in cutaneous leishmaniasis complicated with secondary bacterial infection. Indian J Dermatol 56: 37-39.

Salman S 1999. Cutaneous leishmaniasis: clinical features and diagnosis. Clin Dermatol 17: 291-296.

Scales BS, Huffnagle GB 2013. The microbiome in wound repair and tissue fibrosis. J Pathol 229: 323-331.

Shirazi MH, Ranjbar R, Asgari V, Mohebali M, Hamidian M 2007. Study of bacterial infections among the patients with suspected cutaneous leishmaniasis. Pak J Biol Sci 10: 4555-4558.

Slover CM, Danziger L 2008. Lactobacillus: a Review. Clin Microbiol Newsl 30: 23-27.

Vera LA, de Macedo JLS, Ciuffo IA, Santos CG, Santos JB 2006. Antimicrobial susceptibility of aerobic bacteria isolated from leishmaniotic ulcers in Corte de Pedra, BA. Rev Soc Bras Med Trop 39: 47-50. 
Vera LA, Macêdo VO, de Magalhães AV, Ciuffo IA, Santos CG, Santos JB 2002. Cutaneous leishmaniotic ulcers with Corynebacterium diphtheriae. Rev Soc Bras Med Trop 35: 311-313.

Vieira LQ, Oliveira MR, Neumann E, Nicoli JR, Vieira EC 1998. Parasitic infections in germ-free animals. Braz J Med Biol Res 31: 105-110.

Weirather JL, Jerônimo SMB, Gautam S, Sundar S, Kang M, Kurtz MA, Haque R, Schriefer A, Talhari S, Carvalho EM, Donelson JE, Wilson ME 2011. Serial quantitative PCR assay for detection, species discrimination, and quantification of Leishmania spp in human samples. J Clin Microbiol 49: 3892-3904.

Wolcott RD, Gontcharova V, Sun Y, Zischakau A, Dowd SE 2009. Bacterial diversity in surgical site infections: not just aerobic cocci any more. $J$ Wound Care 18: 317-323.

Ziaei H, Sadeghian G, Hejazi SH 2008. Distribution frequency of pathogenic bacteria isolated from cutaneous leishmaniasis lesions. Korean J Parasitol 46: 191-193. 